\title{
"Curriculitis autoinmune": U na no tan nueva enfermedad del currículo
}

\author{
Sofía P Salas ${ }^{1}$, Ignacio Sánchez $D^{2}$, G uillermo Larios $\mathrm{G}^{3}$, \\ Álvaro Jeria $\mathrm{D}^{4}$, Cristóbal Pertuzé $\mathrm{S}^{\mathrm{a}}$. \\ "Autoimmune curriculitis": \\ A not so new curricular disease
}

Thirty years ago, Abrahamson described the main curriculum problems in undergraduate medical education as "diseases of the curriculum". Based on our own experience and knowledge, we propose the inclusion of a new condition named "autoimmune curriculitis", which is produced by the students themselves. In this condition, the students are who decide to "swell" the curriculum, by excessively participating in multiple intra and extramural activities, competing to markedly improve their academic performance. Unlike other curriculum diseases, this one may directly affect the student's personal life and health, especially if it begins as early as in the first semester of the career. Two main aspects of medical education have a key role in the genesis of this disease: the so-called hidden curriculum and the selection process used in the postgraduate programs in Chile. Unfortunately, the prevalence and severity of this curriculum disease is a growing threat, due to the increasing number of undergraduate students in Chile without an equivalent increment of postgraduate programs. Given its characteristics, eradicating this disease seems quite difficult. To do so, it is necessary for postgraduate selection committees to give a clear sign regarding the most relevant attributes they consider when choosing the candidates and, consequently, to discourage the exaggerated collection of curriculum records. Moreover, we need a group of students faithful to their own interests, who will keep growing in depth those that are more meaningful to them (Rev Méd Chile 2009; 137: 575-81).

(Key words: Curriculum; Education, premedical; Students)

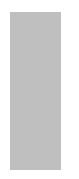

Recibido el 10 de junio, 2008. Aceptado el 27 de octubre, 2008.

Departamentos de ${ }^{1}$ Obstetricia y Ginecología y ${ }^{2}$ Pediatría, ${ }^{3}$ Programa de Formación de Especialistas en Pediatría, ${ }^{4}$ Programa de Formación de Especialistas en Psiquiatría, Escuela de Medicina, Pontificia Universidad Católica de Chile. Santiago de Chile.

aAlumno de Carrera de Medicina. Pontificia Universidad Católica de Chile.

$\mathrm{H}^{\mathrm{s}}$ ace treinta años, y luego de visitar numerosas escuelas de medicina norteamericanas, Abrahamson ${ }^{1}$ describió los principales problemas del

Correspondencia a: Dra. Sofía P. Salas. Departamento de Obstetricia y Ginecología, Escuela de Medicina, Pontificia Universidad Católica de Chile. Marcoleta 391, Santiago. Fono: (562) 354-8172. Fax: (562) 632-1924.

E mail: ssalas@med.puc.cl currículo del pregrado de Medicina, haciendo una analogía con diversas enfermedades. A pesar del tiempo transcurrido, varias de estas alteraciones están aún presentes en la mayoría de las escuelas de medicina chilenas y extranjeras. En este artículo, junto con hacer un breve resumen de las enfermedades descritas por Abrahamson, en base a nuestra experiencia y conocimiento, describimos la existencia de otra enfermedad: la "Curriculitis autoinmune", generada por los mismos estudiantes. 


\section{ANTIGUAS ENFERMEDADES DEL CURRÍCULO}

En primer término, Abrahamson hizo referencia a la "Curriculoesclerosis", producida por una extrema departamentalización, que hace muy rígido el programa de estudios. Esto ocurre cuando los departamentos clínicos ejercen el control sobre los programas de estudio, manteniendo inalterables la importancia relativa de sus respectivas especialidades y la forma en que se entregan los contenidos específicos. El "Carcinoma" del currículo corresponde a un crecimiento descontrolado de un segmento del currículo, sin una lógica enfocada en el aprendizaje del alumno. La "Curriculoartritis" afecta la forma en que segmentos adyacentes del plan de estudio se articulan entre sí, ya sea en forma vertical u horizontal. Así, se observan repeticiones u omisiones no programadas de ciertos contenidos entre un curso y el siguiente, dificultando una adecuada integración de los contenidos. El término "Disestesia" se aplica incluso en aquellos currículos que aparecen diseñados en forma adecuada, pero que al mirar su conjunto, existe la sensación que algo desconocido no anda bien. En la "Curriculitis iatrogénica", son las propias instituciones las responsables de la enfermedad, al estar en forma continua modificando los planes de estudios, sin dar tiempo a entender ni tampoco evaluar los cambios efectuados. La "Curriculomegalia" obedece a la dramática explosión del conocimiento biomédico y a la incapacidad docente para discriminar lo fundamental de lo accesorio; un signo precoz de esta enfermedad es el exceso de horas dedicadas a clases lectivas. En la "Curriculitis idiopática", algo subterráneo no anda bien, por lo que el empleo del tiempo no se relaciona con el aprendizaje. La "Curriculitis intercurrente" puede sobrevenir en forma simultánea con cualquiera de las otras enfermedades y obedece a la incompatibilidad del actual currículo con los problemas sociales que aquejan a la región donde está inserta una escuela de medicina. La última enfermedad descrita fue la "Osificación Curricular", en la cual el currículo es tan rígido que aparece como imposible de cambiar. La tradición, el "siempre se ha enseñado asi", o "así aprendí yo" tienen más fuerza que la evidencia otorgada por la medicina moderna.

La génesis de estas enfermedades se asocia a una relativa incapacidad de los comités de currí- culo: éstos no tienen el poder necesario para estructurar un plan de estudios coherente y supervisar su implementación o, por el contrario, realizan cambios sucesivos sin darse el tiempo para evaluar su impacto en las competencias de los egresados. En todos estos casos, los estudiantes experimentan los signos y síntomas de estas enfermedades, pero no son responsables de su génesis ni de su tratamiento.

\section{CURRICULITIS AUTOINMUNE}

Proponemos la existencia de otra enfermedad del currículo: la "Curriculitis autoinmune". En ésta, son los propios estudiantes los que "inflaman" el currículo, al aumentar en forma exagerada su participación en múltiples actividades, tanto intra como extramurales, compitiendo por mejorar su rendimiento académico en forma desmedida. A diferencia de las anteriores enfermedades curriculares, ésta impacta directamente en la vida personal y en la salud de los estudiantes, en especial si comienza precozmente.

No nos referimos a la participación en deportes, en el grupo de teatro, en la pastoral o en actividades sociales, sino que a la búsqueda frenética de actividades que contribuyan a incrementar las posibilidades de acceder a un programa de postítulo. Esta "inflamación del currículo" no siempre busca un mejor desempeño profesional futuro, sino que muchas veces carece de una justificación o coherencia con los intereses, valores e ideales del estudiante.

Dependiendo de la severidad de esta enfermedad, los estudiantes pueden llegar a renunciar a las actividades que realizaban antes de ingresar a la escuela de medicina y que pudiesen interferir con su desempeño académico. Es necesario diferenciar entre la necesidad de abandonar actividades de desarrollo personal si el estudiante está apremiado por un bajo rendimiento, versus el abandonar sus intereses en pos de aumentar artificialmente sus logros académicos.

\section{ETiopatogenia DE la CURRICULITIS AUTOINMUNE}

En la génesis de esta enfermedad, participarían principalmente dos aspectos fundamentales de la 
formación médica: el denominado currículo oculto, presente en las diversas instituciones encargadas de dicho proceso, y el sistema de selección para los programas de postgrado en Chile.

En relación al primer punto, Coulehan, en su artículo sobre profesionalismo, describió un conflicto entre aquello que creemos estar enseñando (currículo explícito o formal) y aquello que los estudiantes aprenden a partir de otras fuentes (currículo tácito, informal u oculto) ${ }^{2}$. Este conflicto, que comienza precozmente, se acentúa al llegar a la clínica y al internado y se sigue desarrollando durante la especialización. Mientras el currículo explícito se enfoca en la empatía, comunicación, alivio del sufrimiento, confianza y fidelidad, teniendo al paciente en el centro de la preocupación, el currículo oculto induce a los estudiantes a no involucrarse, a esconder sus emociones, y principalmente a centrarse en sus propios intereses. Así, lo que los estudiantes ven hacer a sus docentes difiere de lo que les escuchan decir $^{2}$. Esto mismo puede extenderse a lo observado en los procesos de selección al postítulo: mientras las autoridades académicas preconizan privilegiar los valores, el altruismo y el trato compasivo, al seleccionar a los becados según la mirada de los alumnos- parecieran privilegiarse aspectos como el rendimiento académico (notas y calificación en el Examen Médico Nacional) y los trabajos de investigación, por sobre los valores personales. Pero lo anterior no explica el fenómeno en su totalidad. El currículo oculto se expresa, además, en el contacto de los estudiantes con sus pares de cursos superiores, quienes transfieren parte de este modelo adquirido a los alumnos nuevos. Como fue explicitado en el informe que los estudiantes de la Escuela de Medicina de la Pontificia Universidad Católica de Chile (PUC) hicieron durante el proceso de autoevaluación realizado el año 2007, con motivo de una visita de la American Association of Medical Colleges, el estudiante actuaría según "presiones del ambiente" y de sus mismos compañeros, modificando su experiencia de vida universitaria en pos de "mejores antecedentes". Esto favorecería "un clima de competitividad a ultranza entre los estudiantes, dejando de lado valores esenciales como el compañerismo y la mutua cooperación"3,4. Otros autores han sugerido que durante sus estudios médicos, los estudiantes pierden varios de los atributos considerados como deseables, pasando de ser personas intelectualmente curiosas a estar enfocados en conocimientos y competencias específicas para pasar un examen, de ser empáticos a estar emocionalmente protegidos, de ser idealistas a ser cínicos sobre la práctica médica $^{5}$. Tal como lo menciona Glicken, esta declinación en los valores ideales no sería consecuencia de omisiones en el currículo formal, sino que a la impronta que confiere el currículo oculto. Así, lo que los estudiantes observan en el día a día tendría una influencia mucho más importante que lo enseñado en los cursos formales.

\section{SínTomas y SIGNOS DE LA CURRICUUTTS AUTOINMUNE}

Esta enfermedad se expresa en diversos grados y formas entre los estudiantes de medicina. Se caracteriza por la realización de actividades extracurriculares que son percibidas por ellos como importantes en el proceso de selección al postítulo y fomentadas por este ambiente de competencia. En una reciente encuesta realizada a internos de $7^{0}$ año de la PUC, 69/76 (91\%) de los estudiantes participaron en un proyecto de investigación, y 46/76 (61\%) fueron autores de al menos un trabajo de investigación durante sus años de estudio. Este fenómeno no es exclusivo de los graduados de la PUC, puesto que en los currículos de los postulantes a becas de especialización en la PUC, provenientes de distintas escuelas de medicina chilenas, se observa un fenómeno parecido. Cabe destacar que durante el postítulo, estos becados no mantienen este ritmo de productividad científica, medida en proyectos o publicaciones, porque su interés no estaba puesto en la investigación.

Es común también que los estudiantes afectados por curriculitis autoinmune presenten conductas de deshonestidad académica, como la copia en pruebas y exámenes, tal como se observó en la encuesta del proceso de autoevaluación realizada el año $2007^{3}$ y al aplicar la encuesta DREEM, realizada por Riquelme y colaboradores a estudiantes entre $3^{0}$ y $5^{0}$ año (comunicación personal); en ambos casos los estudiantes señalaron que el ambiente de competitividad promueve este tipo de conducta. A diferencia de lo que ocurre en otras carreras, la justificación de la copia 
estaría en aumentar el rendimiento académico, y no en evitar reprobar el ramo. Una encuesta a cerca de 2.500 estudiantes de medicina norteamericanos, mostró que 5\% reportó haber copiado personalmente, mientras que casi $40 \%$ señaló haber visto a uno de sus compañeros copiar ${ }^{6}$, lo que refleja que es un problema generalizado. Otro estudio evaluó los cambios de percepción de los estudiantes con respecto a la potencial deshonestidad de determinadas conductas, determinando que a medida que se progresaba en la carrera, más estudiantes no tenían reparos en falsificar la firma de un docente o en describir como "normal" el examen físico de un paciente, cuando en realidad éste no había sido realizado ${ }^{7}$. Si bien es imposible precisar las causas de este cambio en la percepción de lo que es deshonesto, los autores plantean que esto puede deberse a presiones extrínsecas por aumento de la carga académica y también a presiones intrínsecas debido a una mayor sensación de pérdida frente a un potencial fracaso. Esto está también presente en nuestros alumnos, en especial al formar los grupos de internado, cuando se observa que por sobre la amistad y afinidad, se privilegia formar grupos con estilos de trabajos similares que aseguren un mejor desempeño.

Llama la atención que la gran mayoría de los estudiantes evalúa en forma negativa la cantidad y calidad del tiempo libre durante su paso por el pregrado. Este es considerado como escaso y suele estar mal distribuido a lo largo del currículo, siendo especialmente crítico en nuestra Escuela en el internado de sexto año. Parte de esta sobrecarga corresponde efectivamente a planes de estudio sobredimensionados, que no dan lugar a tiempo libre o de estudio personal. Otra parte importante se debe a la actitud de los mismos estudiantes, que es lo que hemos designado como "curriculitis autoinmune". Esto fue recogido en el Claustro de Estudiantes realizado el año 20028, donde en un grupo focal los estudiantes señalaron que percibían una baja calidad de vida, atribuible a las altas demandas académicas dentro de la carrera y también a los niveles de autoexigencia, que los llevaba a aumentar en forma desmedida la jornada de trabajo, más allá de las exigencias propias de las rotaciones clínicas. Por otra parte, destaca el hecho de que los alumnos no utilizan apropiadamente el tiempo cuando lo pueden hacer, ya que no realizan mayores actividades extraprogramáticas en el internado de $7^{\circ}$ año, que en la PUC corresponde a un período electivo en el cual pueden elegir libremente las rotaciones más afines a sus áreas de interés.

Esta patología es crónica y de evolución progresiva. En el tramo final, ad portas del proceso de selección a los programas de especialización, el cuadro se observa con una gravedad a veces inusitada. La primera preocupación del estudiante afectado por curriculitis será conseguir la mayor cantidad de publicaciones, diplomas y certificados, para así abultar sus antecedentes académicos. Afortunadamente, existen algunos estudiantes protegidos de esta enfermedad, logrando mantenerse ajenos a la curriculitis a pesar de presenciar su propagación entre sus pares.

\section{ENFERMEDADES ASOCIADAS A LA CURRICULTIS}

Este nivel de competitividad -que no es exclusivo de los estudiantes de medicina, sino que también refleja la competitividad de la sociedad en general- ha sido considerado como el estímulo para el desarrollo de otras enfermedades. Diversos estudios han mostrado que los estudiantes de medicina presentan con frecuencia morbilidad psiquiátrica y altos niveles de estrés ${ }^{9,10}$, muchas veces asociados a sobrecarga de trabajo. Por desgracia, la mayoría de los estudiantes no buscan ayuda oportuna, por considerar que pueden quedar estigmatizados, dificultando su progresión académica y la obtención de becas y residencias $^{11}$. Un estudio norteamericano reportó la presencia de "burnout" en $45 \%$ de los estudiantes de medicina, el cual aumentaba en alumnos de cursos superiores debido a mayor carga académica y también a factores personales estresantes ${ }^{12}$. Algo similar se observó en estudiantes de Turquía, en quienes un aumento de eventos vitales adversos, tales como insatisfacción por las actividades sociales, preocupación por el futuro y temor al fracaso en los exámenes, se asoció a una disminución en indicadores de bienestar de salud ${ }^{13}$. Estos hallazgos sugieren que tanto factores curriculares como personales deben tomarse en cuenta para intentar disminuir el "burnout" de los estudiantes. Cabe señalar que el desempeño de los estudiantes universitarios es en sí mismo un factor desencade- 
nante de estrés psicológico, lo que incide en presentar cifras de morbilidad siquiátrica superiores a las de la población general ${ }^{14}$. La situación se vuelve aún más preocupante si consideramos que estudios recientes muestran que hasta un tercio de la población chilena relata haber tenido algún trastorno psiquiátrico al menos una vez en sus vidas $^{15}$, mientras que los resultados de la última encuesta nacional de salud reportan que 17,5\% de los encuestados tuvo síntomas depresivos en el último año ${ }^{16}$. En estudiantes de medicina de la PUC se evaluó el riesgo de padecer patología psiquiátrica mediante la aplicación del General Health Questionnaire 12 item (prueba de Goldberg abreviada), demostrando que $41 \%$ de los estudiantes entre $1^{0}$ y $5^{0}$ año tenían Goldberg $(+)^{17}$, considerado como riesgo de patología mental. Esto es concordante con otros estudios que muestran alta prevalencia de patología mental en estudiantes de medicina. Así, un estudio noruego mostró que $31 \%$ de los estudiantes de medicina requirieron atención especializada por problemas de salud mental en los primeros tres años de la carrera ${ }^{18}$, mientras que otras series han reportado presencia de morbilidad psicológica entre $20 \%$ a $44 \%$ de los estudiantes de medicina ${ }^{19-21}$.

En el Claustro del año 2002, los estudiantes señalaron que la alta carga académica se reflejaba en una baja calidad de vida, la cual influía en sus relaciones interpersonales, en su vida afectiva y familiar y también frenaría sus posibilidades de realizar actividades extraacadémicas y de aprovechar otros ámbitos de la vida universitaria. Tal como señalaron los estudiantes, la "ausencia de tiempo no lectivo... impide que el alumno pueda desarrollar a cabalidad su formación de médico integral"3. Sería importante repetir estas encuestas, ya que en este lapso de 5 años se han realizado varios cambios en el currículo de nuestra Escuela de Medicina, por lo que estas percepciones podrían haberse modificado.

\section{PRONÓSTICO Y TRATAMIENTO}

Lamentablemente, esta enfermedad curricular amenaza con aumentar su prevalencia y gravedad, debido al importante crecimiento de alumnos de pregrado de Medicina en Chile, sin un correspondiente aumento en programas de especialización.
Si en la actualidad aproximadamente 900 egresados compiten por un cupo de los 660 ofrecidos en especialidades primarias, en un futuro no muy lejano el número de egresados casi triplicará la oferta de cupos, por lo que los postulantes se enfrentarán a una competencia aún más férrea.

Uno de los elementos que destaca en esta conducta desondenada y sin un objetivo claro, es la inseguridad de los alumnos en su propio proyecto personal. Es importante reafirmarles que sólo el trabajo dedicado, a conciencia, con un conocimiento y experiencia profundos y sólidos, será capaz de generar nuevo conocimiento, cambiar conductas y, finalmente, tener impacto en la sociedad. La mayoría de los trabajos que se realizan sólo para sumar puntos, suelen ser breves y superficiales y no tendrán una consecuencia positiva para la carrera del estudiante e incluso son considerados con reserva por las comisiones de selección de alumnos de postgrado, puesto que no proyectan una imagen coherente con respecto a los intereses del postulante. Algo semejante sucede con algunas actividades extraprogramáticas que son realizadas con el solo objeto de ampliar el currículo.

Parece difícil disminuir el estrés que experimentan los estudiantes de medicina sin que se modifiquen sus causas, las que son de origen diverso. Experiencias internacionales sugieren, sin embargo, que la realización de "electivos" dirigidos a disminuir el estrés o a promover el bienestar pueden tener efectos positivos que perduran al menos en el corto plazo 22,23 . Asimismo, contribuye al éxito académico la implementación de un diseño curricular y un ambiente de aprendizaje centrado en los estudiantes, lo que ha cambiado el foco de una "patología de la educación médica" a una educación médica que promueve el estado de salud de sus estudiantes al lograr un adecuado equilibrio entre las altas demandas inherentes a la educación médica con una vida plena fuera de las aulas universitarias ${ }^{24}$. Otro programa que ha mostrado resultados positivos a lo largo del tiempo, consiste en un sistema de tutonías de alumnos de $2^{\underline{ }}$ año a los de 10, permitiendo que los estudiantes comprendan las potenciales fuentes de estrés al comienzo de la carrera, a partir de modelos considerados como cercanos y creíbles. Esta experiencia es también beneficiosa para los alumnos mayores, puesto que adquieren confianza en sus habilidades para escuchar con empatía y lograr relajar a sus futuros pacientes $^{25}$. Otra iniciativa que puede ayudar a 
modificar estas conductas es la implementación de tutorías en las cuales un grupo pequeño de alumnos es guiado por docentes de la Escuela de Medicina durante los primeros años de la carrera, experiencia que ha mostrado ser exitosa para todos los involucra$\operatorname{dos}^{26}$ y que se ha implementado en el último año en nuestra Escuela. De esta forma se espera que el modelaje y enseñanza de roles sea orientado a los valores que enfaticen la comunicación y la entrega personal al paciente, más que a la búsqueda del beneficio personal por parte del alumno.

Por lo tanto, como sugiere Kenny, es necesario enfocar nuestra atención no tanto en los individuos, sino más bien en la institución y su modelaje de valores ${ }^{27}$, puesto que se requiere de una postura filosófica institucional que sea coherente con las estrategias educacionales específicas y que promueva el profesionalismo como un aspecto central de la práctica médica. Por último, hay que tener presente que el problema de la alta competitividad que presentan los estudiantes de medicina es un fenómeno sociológico que abarca toda nuestra cultura, por lo que parece difícil que las escuelas de medicina, sus directivos y los estudiantes se puedan sustraer a esta realidad.

\section{REFERENCIAS}

1. Abrahamson S. Diseases of the curriculum. J Med Educ 1978; 53: 951-7.

2. Coulehan J. Viewpoint: today's professionalism: engaging the mind but not the heart. Acad Med 2005; 80: 892-8.

3. Larios G, Pertuzé CF, Aguirre D, Astudilo S, Herrera C, Jeria A. Proceso de Acreditación AAMC 2007. Análisis estudiantil de la Escuela de Medicina de la Pontificia Universidad Católica; 2007.

4. JeRIA A. Currículo oculto en la carrera de medicina: percepción desde una experiencia estudiantil. En: Mena B, editor. IV Congreso de Educación Médica. Ensayos Finalistas; 2007; Santiago, Chile: Pontificia Universidad Católica de Chile; 2007; 25-30.

5. GLicken AD, Merenstein GB. Addressing the hidden curriculum: understanding educator professionalism. Med Teach 2007; 29: 54-7.

6. Baldwin DC Jr, Daugherty SR, Rowiey BD, Schwarz MD. Cheating in medical school: a survey of second-year students at 31 schools. Acad Med 1996; 71: 267-73.

7. RENNIE SC, Rudiand JR. Differences in medical students' attitudes to academic misconduct and reported behavior across the years-a questionnaire study. J Med Ethics 2003; 29: 97-102.

\section{CONCLUSIONES}

Treinta años después que Abrahamson describiera las nueve enfermedades del currículo, es posible observar que varias persisten en diversas escuelas de medicina. Por lo tanto, parece difícil pensar que se pueda erradicar la "curriculitis autoinmune”. Para esto, se requeriría una señal clara por parte de los comités de selección al postítulo, con respecto a qué atributos consideran los más relevantes a la hora de seleccionar a los candidatos a beca, desincentivando la recolección desmedida de antecedentes curriculares. Para que esta medida tenga algún impacto, debiera ser conocida por todos los involucrados. Asimismo, se necesita de un grupo de estudiantes fieles a sus propios intereses y que cultiven en profundidad aquello que les hace más sentido. Queremos que sólo investiguen los estudiantes con real vocación científica, que sólo realicen trabajo comunitario aquellos que tienen vocación de ayuda social y que todos, en forma coordinada, decidan dedicar parte de su escaso tiempo libre a que éste siga siendo "tiempo libre" y de ocio para actividades familiares, deportivas, artísticas y culturales.

8. Escuela de Medicina de la Universidad Católica. Características del perfil de médico esperado por la escuela de medicina de la Pontificia Universidad Católica de Chile; Documento de Trabajo Escuela de Medicina de la Universidad Católica de Chile, 2002.

9. Dahin M, Joneborg N, Runeson B. Performancebased self-esteem and burnout in a cross-sectional study of medical students. Med Teach 2007; 29: 43-8.

10. DAhun ME, Runeson B. Burnout and psychiatric morbidity among medical students entering clinical training: a three year prospective questionnaire and interview-based study. BMC Med Educ 2007; 7: 6 .

11. Chew-Graham CA, Rogers A, Yassin N. 'I wouldn't want it on my CV or their records': medical students' experiences of help-seeking for mental health problems. Med Educ 2003; 37: 873-80.

12. Dyrbye LN, Thomas MR, Huntington JL, Lawson KL, Novotny PJ, SLoan JA, Shanafelt TD. Personal life events and medical student burnout: a multicenter study. Acad Med 2006; 81: 374-84.

13. Aktekin M, Karaman T, Senol Yy, Erdem S, Erengin H, AkAYDin M. Anxiety, depression and stressful life events among medical students: a prospective study in Antalya, Turkey. Med Educ 2001; 35: 12-7. 
14. Staluman HM. Prevalence of psychological distress in university students - Implications for service delivery. Aust Fam Physician 2008; 37: 673-7.

15. Vicente B, Rioseco P, Saldivia S, Kohn R, Torres S. Estudio chileno de prevalencia de patología psiquiátrica (DSM-III-R/CIDI) (ECPP). Rev Med Chile 2002; 130: 527-36.

16. Encuesta Nacional de Salud Chile 2003. Ministerio de Salud. Disponible en http://epi.minsal.cl [consultado el 22 octubre 2008].

17. Benítez C, Quintero J, Torres R. Prevalencia de riesgo de trastornos psiquiátricos en estudiantes de pregrado de la Escuela de Medicina de la P. Universidad Católica de Chile. Rev Med Chile 2001; 129: 173-8.

18. Midtgaard M, Ekeberg O, Vaglum P, Tyssen R. Mental health treatment needs for medical students: a national longitudinal study. Eur Psychiatry 2008; 23: 505-11.

19. SReeramareddy CT, Shankar PR, Binu VS, Mukhopadhyay C, Ray B, Menezes RG. Psychological morbidity, sources of stress and coping strategies among undergraduate medical students of Nepal. BMC Med Educ 2007; 7: 26.

20. Carson AJ, Dias S, Johnston A, McLoughun MA, O'ConNor M, Robinson BL ET AL. Mental health in medical students. A case control study using the 60 item General Health Questionnaire. Scott Med J 2000; 45: 115-6.

21. Assadi SM, Nakhaei MR, Najafi F, Fazel S. Mental health in three generations of Iranian medical students and doctors. A cross-sectional study. Soc Psychiatry Psychiatr Epidemiol 2007; 42: 57-60.

22. Finkelstein C, Brownstein A, Scott C, Lan YL. Anxiety and stress reduction in medical education: an intervention. Med Educ 2007; 41: 258-64.

23. Lee J, Graham AV. Students' perception of medical school stress and their evaluation of a wellness elective. Med Educ 2001; 35: 652-9.

24. Kiessing C, Schubert B, Scheffner D, Burger W. First year medical students' perceptions of stress and support: a comparison between reformed and traditional track curricula. Med Educ 2004; 38: 504-9.

25. REDwood SK, PoшaK MH. Student-led stress management program for first-year medical students. Teach Learn Med 2007; 19: 42-6.

26. WiLSON LD. Faculty mentorship and first year students at the Yale School of Medicine: reflections and perspective. Yale J Biol Med 2004; 77: 155-7.

27. KenNy NP, ManN KV, MacLeod H. Role modeling in physicians' professional formation: reconsidering an essential but untapped educational strategy. Acad Med 2003; 78: 1203-10. 